\title{
GEOSYNCHRONOUS SATELLITE ORBIT \\ DETERMINATION
}

\author{
SERGEI RUDENKO \\ Main Astronomical Observatory, National Acad. of Sciences \\ Golosiiv, Kiev, Ukraine
}

\begin{abstract}
The paper presents the results of the orbit determination of geosynchronous satellites derived from 1921 positional observations of a few dozens of satellites obtained at the double wide-angle astrograph DWA $(\mathrm{D}=0.4 \mathrm{~m}, \mathrm{~F}=2 \mathrm{~m})$ of the Main Astronomical Observatory of the National Academy of Sciences of Ukraine in 1985-1991. The observations cover the longitude range from $19^{\circ} \mathrm{W}$ to $80^{\circ} \mathrm{E}$. Orbital inclination of the observed objects is less than $3^{\circ}$. The mean values of the rms residuals are 0.66 in R.A. and 1".01 in Dec. over all processed orbital arcs.
\end{abstract}

\section{Introduction}

Geosynchronous satellites are widely utilized for communication, meteorological surveillance and ground control purposes. They are observed from the Earth by passive sensors such as radar and optical devices. The number of geostationary and near-geostationary objects catalogued by the ESA/ESOC DISCOS Database is 603 on February 8, 1996 (Log of objects, 1996). Moreover, their number is increased by 30-35 objects per year. Operational satellites are frequently manoeuvred by Earth-based control centers to prevent their running away from the assigned longitude window. Thus, the problem of geosynchronous objects orbit determination and identification is continuous.

Using the software developed at the Main Astronomical Observatory of the National Academy of Sciences of Ukraine (GAOUA) to analyze the positional observations of geosynchronous satellites (Rudenko, 1995) we processed the photographic observations of geosynchronous satellites obtained at GAOUA in 1985-1991 (Kizyun et al., 1995) for scientific and applied studies. The paper presents the results of this analysis.

I. M. Wytrzyszczak, J. H. Lieske and R. A. Feldman (eds.), Dynamics and Astrometry of Natural and Artificial Celestial Bodies, 351, 1997.

(C) 1997 Kluwer Academic Publishers. Printed in the Netherlands. 


\section{Data Analysis}

\subsection{THE MAIN STEPS OF THE ORBIT DETERMINATION PROCEDURE}

The input data for the analysis is the following:

- a catalogue of positional observations of satellites, i.e. topocentric right ascension (R.A.) and declination (Dec.) referred to either the true-ofdate, or mean, or B1950.0, or J2000.0 reference system;

- geocentric Cartesian station coordinates represented in the Terrestrial Reference Frame (TRF).

The data processing is performed in the following steps:

1. Satellite observations are transformed to the J2000.0 reference frame being accepted as the standard one.

2. The initial value of the satellite state-vector is determined under the circular orbit approximation using the minimum required information: two observations at each orbital arc.

3. The orbit prediction procedure is performed using the precise algorithm ("Kiev-Geodynamics-G" software) to calculate the following parameters at the instant of each observation:

- satellite coordinates in the Celestial Reference Frame (CRF);

- station coordinates in the CRF;

- $(\mathrm{O}-\mathrm{C})$ residuals in R.A. and Dec.;

- partial derivatives of the observed R.A. and Dec. with respect to the satellite state-vector.

4. The orbit improvement procedure is realized by the differential improvement of the satellite state-vector at the beginning of each orbital arc by the least square adjustment.

5 . The steps 3 and 4 are repeated until convergence.

6. A special analysis has to be performed in the case when the resulting rms residuals either in R.A. or Dec. are large, i.e. more than a few arc seconds. It can be due to either an error in the input observations, or a satellite orbital manoeuvre performed at the processed orbital arc. In the latter case the initial orbital arc is divided into sub-arcs which are treated separately.

The output of the orbit determination procedure is a catalogue containing the information as follows:

- the state-vector, keplerian elements (both in the CRF), longitude and latitude in the TRF of each satellite at the beginning of each orbital arc etc.;

- statistical information of the orbit improvement procedure (rms values of the post-fit residuals in R.A. and Dec.). 


\section{2. "KIEV-GEODYNAMICS-G" SOFTWARE MAIN CHARACTERISTICS}

The software is based on numerical integration. The code VASOMI realizing the variable order, variable step size Adams method is used. The reference frame transformation is performed as recommended by the IERS Standards (McCarthy, 1992) using the IAU 1976 precession model, the IAU 1980 nutation theory with the celestial pole-of-sets from the EOP (IERS) C04 series, and the Earth rotation parameters from the same series.

In the present analysis the following force model was used:

- the Earth's gravity field (JGM-3 model) up to $n=m=6$;

- third-body gravitational attraction by the Moon, the Sun and the major planets Venus and Jupiter (LE200/DE200 ephemerides);

- solar radiation pressure (when $\mathrm{A} / \mathrm{m}$ is known).

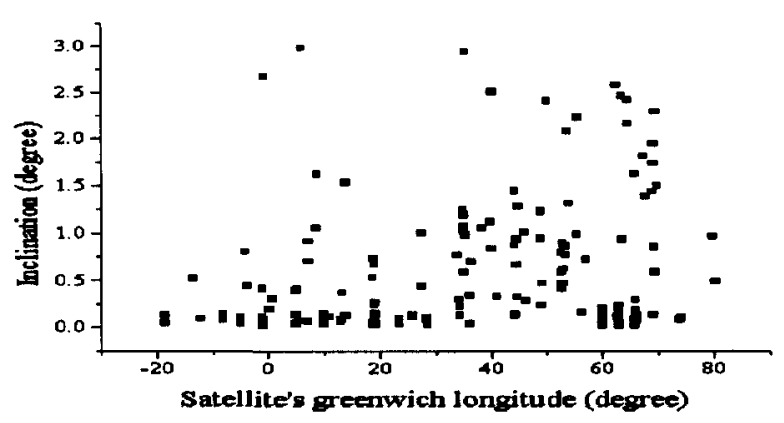

Figure 1. Distribution of observed satellites by longitude and inclination.

\section{Results}

We processed 1921 observations of geosynchronous satellites obtained at the time span from May 12, 1985 to October 8, 1991. These observations correspond to 525 orbital arcs. An orbital arc means the observations of some object at some time span. Usually, there are observations of some satellite at a few orbital arcs. We managed to calculate the reliable orbits for 214 orbital arcs with the number of observations superior or equal to 4. Figure 1 presents the distribution of the satellites corresponding to the orbital arcs by longitude $\lambda$ and inclination $i$.

It turned out difficult to determine the orbits of the objects with $i>3^{\circ}$ since for these objects only 1 or 2 positions were detected on the plates during the surveys.

As a rule, it was sufficient to perform 2-3 iterations of the orbit adjustment procedure at each orbital arc until convergence. The graphs of rms values of the post-fit $(\mathrm{O}-\mathrm{C})$ residuals in R.A. and Dec. at the processed orbital arcs are shown in Figures 2 and 3, respectively. 


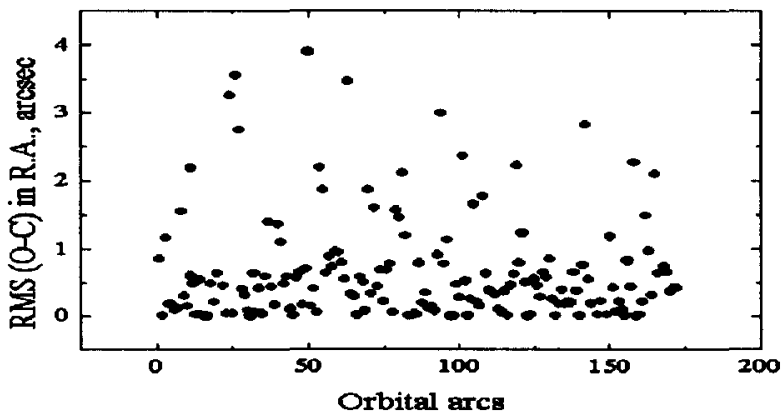

Figure 2. Rms values of the (O-C) residuals in R.A. at the orbital arcs.

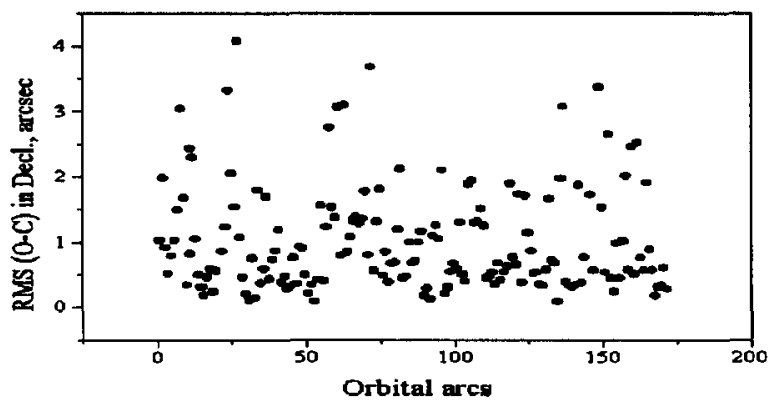

Figure 3. Rms values of the $(\mathrm{O}-\mathrm{C})$ residuals in Dec. at the orbital arcs.

The mean values of the rms residuals are 0"66 in R.A. and 1".01 in Dec. over all processed orbital arcs. The cases when orbital manoeuvres took place are not shown on these graphs, since the rms residuals reached a few arc minutes at these arcs.

The resulting catalogue of the satellite state-vectors and Keplerian elements can be used in satellite identification and orbit propagation.

Acknowledgements. The participation of the author at the Colloquium was supported by the IAU travel grant which is greatly acknowledged.

\section{References}

Kizyun, L.N. and Safronov, Yu. I.: 1995, Catalogue of Positions of the Geostationary and Quasi-Geostationary Earth Satellites, Preprint, Main Astronomical Observatory, National Academy of Sciences of Ukraine, Kiev, 121.

Log of Objects Near the Geostationary Ring, Issue 16, ESA/ESOC. February 1996.

McCarthy, D. D., (ed.): 1992, "IERS Standards (1992)", IERS Tech. Note 13, Obs. de Paris.

Rudenko, S.: 1995, "Software for the analysis of photographic observations of geosynchronous satellites", in: Accurate Orbit Determination and Observations of High Earth Satellites for Geodynamics, COGEOS Workshop in Walferdange (A. Elipe, P. Paquet, eds), Luxembourg, 63-70. 